\title{
Detection of Malicious Attacks in Autonomous Cyber-Physical Inverter-Based Microgrids
}

\author{
Ioannis Zografopoulos, Graduate Student Member, IEEE, Charalambos Konstantinou, Senior Member, IEEE
}

\begin{abstract}
The distributed generation capabilities of microgrids (MGs) arise as essential assets in enhancing grid resilience. The integration of distributed energy sources (DERs), controllable loads, and prosumers necessitates the deployment of potent control and communication synergies. While those synergies transform MGs into cyber-physical systems through information technologies able to sense, control, and actuate local resources and loads, they inadvertently expose MGs to cyber-attack threats. Increasing the security of critical communication and control systems against 'black swan' events, i.e., high-impact lowprobability cyber-physical incidents, is a major priority for MGs operations. Such incidents, if left unabated, can intensify and elicit system dynamics instability, eventually causing outages and system failures. In this paper, we develop an integrated approach for multi-agent MG systems able to perform the detection of malicious cyber-physical attacks based on subspace methods. We employ the small signal model of an autonomous/islanded MG and consider different attack models targeting the MG's secondary frequency control. The attack detector is constructed via identifying the stable kernel representation of the autonomous cyber-physical MG in the attack-free case. We illustrate the impact of the attack models as well as the feasibility of the developed detection method in simulation models of the Canadian urban benchmark distribution system.
\end{abstract}

Index Terms-Cyber-physical microgrids, attacks, detection.

\section{INTRODUCTION}

In recent years, power grids have undergone radical changes to account for the increasing energy demands, satisfy environmental regulations, and improve power quality and system resilience. Furthermore, the unprecedented energy demand during COVID-19 underlined the obstacles that future power systems - dealing with the stochasticity of power demand profiles, and integrating renewable and/or distributed resources with their inherent voltage, and power quality characteristics - will have to overcome. Microgrids (MGs) are critical to help solve such issues with smart controls, distributed energy resources (DERs) management, and coordination with the grid and customers. MGs' autonomous operation and ancillary services can bolster grid resilience by supporting missioncritical organizations (e.g., healthcare facilities), and maintaining power supply during natural disasters or extreme weatherrelated events (e.g., like the 2021 winter storm in Texas).

MGs have slowly started to transform into cyber-physical MGs incorporating decentralized and distributed multi-agent frameworks and integrating advanced power electronics as well as a growing number of embedded devices for voltage and power factor control [1]. All these agents are connected through the physical layer representation of the system (e.g.,

The authors are with the Computer, Electrical and Mathematical Sciences and Engineering (CEMSE) Division, King Abdullah University of Science and Technology (KAUST), Thuwal 23955-6900, Saudi Arabia.

E-mail: \{firstname.lastname\}@kaust.edu.edu. power lines), and on top of the physical nodes and links, the cyber information and communication technology (ICT) network connects the different cyber-physical components. The inclusion of ICT as part of their control and telemetry functions is also accompanied by important technical challenges [2], [3]. Among them, cybersecurity is of paramount importance as adverse cyber-attack incidents of sophisticated and destructive nature are growing unabated, especially at the distribution grid level [4].

To address disruptive attacks in MGs, in this work, we devise realistic attack strategies within a cyber-physical MG and propose an attack detection framework as well as assess the system-level effects of attacks on MGs operation. Specifically, we investigate the behavior of interconnected and islanded inverter-based cyber-physical MG systems under three attack models. Such attacks are captured in a stochastic monolithic system model, which in contrast with literature, does not require full knowledge of the system characteristics. We evaluate the MG operation considering the occurrence of noises, and demonstrate the attack detectable conditions, with the aid of subspace identification, that address such disruptive attacks in order to promptly avoid cascading effects, and hence enhance system resilience.

The roadmap of the paper is organized as follows. Section IIII describes the inverter-based MG architecture and the system dynamics model. Section IV presents the proposed attack formulation and threat model. Section $\mathrm{V}$ demonstrates the novel detection framework and Section $\mathrm{VI}$ provides the simulation case studies which examine the system resilience in the presence of attacks. Section VII concludes the paper.

\section{RELATED WORK}

In the literature, work on securing cyber-physical systems extends into two broad dimensions: red-team type of attack evaluation methods, which aim is to create challenging and realistic test scenarios to identify vulnerabilities and evaluate the security posture of the system, and blue-team type of attack detection and mitigation mechanisms, which, based on the results of the attacks evaluation, implement countermeasures able to detect and minimize the blast radius of the attacks. In Table I we present a variety of research studies aiming to enhance cyber-physical MG security leveraging diverse approaches for the attack detection, modeling, risk evaluation and mitigation. Applying such methods in cyber-physical MGs leads to several challenges given their service criticality and real-time characteristics; existing research on attack evaluation considers only a specific attack model that requires substantial knowledge of the MG system and controls, without being able to capture multi-variate attack characteristics [5], [16], 
Table I: Related literature for cyber-physical microgrid (MG) security.

\begin{tabular}{|c|c|c|}
\hline Study Type & Methodology & Ref. \\
\hline \multirow{6}{*}{$\begin{array}{c}\text { Attack } \\
\text { Detection }\end{array}$} & Artificial intelligence-based detection of attacks in DC MGs. & {$[5]$} \\
\hline & False data detection using wavelet transform and neural networks. & {$[6]$} \\
\hline & False data detection using Go Decomposition. & {$[7$} \\
\hline & Data integrity pattern recognition using spectral and wavelet distributions. & {$[8$} \\
\hline & False data detection leveraging physical invariants of DC MGs & {$[\overline{9}$} \\
\hline & Centralized and distributed attack identification. & 10 \\
\hline \multirow{2}{*}{$\begin{array}{l}\text { Modeling and } \\
\text { Simulation }\end{array}$} & Framework for modeling, simulation and impact assessment of cyber-attacks. & $\overline{11}$ \\
\hline & $\begin{array}{l}\text { High performance computing for power system analyses. } \\
\end{array}$ & $\overline{12}$ \\
\hline \multirow{2}{*}{ Attack Impact } & Impact of communication delays on islanded MGs. & [13 \\
\hline & False data injection attack impact on autonomous MGs. & $\overline{14}$ \\
\hline \multirow{4}{*}{$\begin{array}{c}\text { Attack } \\
\text { Mitigation }\end{array}$} & Impact of time-varying attacks and distributed control-based mitigation. & $\overline{15}$ \\
\hline & Event-driven strategy for the replacement of attacked signals. & $\overline{16}$ \\
\hline & Sensor attack-resilient output-feedback controller design. & $\overline{17}$ \\
\hline & Corrupted measurement-agnostic observer design. & $\overline{18}$ \\
\hline \multirow{3}{*}{$\begin{array}{l}\text { Detection and } \\
\text { Mitigation }\end{array}$} & MG DoS attack detection and mode-dependent controller design. & $\overline{19}$ \\
\hline & Resilient state estimation under sensor attacks. & $\overline{20}$ \\
\hline & Data-driven approaches for anomaly detection and controller design. & $\overline{21}$ \\
\hline
\end{tabular}

involving the adversary's capabilities, intentions, and objectives within a MG as well as how to model attacks based on their specific methodology, targeted system component, and system impact, as well as define rules that enable multi-layer and severity attack analyses [11]. Liu et al. consider attackers with full access to communication signals of MGs able to cause delays [13] and denial-of-service (DoS) attacks [19]. Similarly, Zhou et al. assume attacks on MG communications links as well as local and master controllers where adversaries could interrupt any signal with full knowledge and access [15]. In addition, the false data injection attack strategy developed in [14] assumes full knowledge of the power system with an adaptive offline adversary (strong adversary) who knows everything. In this work, the attacker model, considering the unique characteristics of MGs and their operation environment, adopts a realistic approach which does require full knowledge of the system topology, lines parameters, and component interconnection details. Our adversary model assumes access to the metering infrastructure allowing the estimation of plausible attack vectors based on historical measured data. We build a stochastic monolithic system model which can capture attacks on measurements, model inputs, and load profiles.

On the other hand, existing attack detection schemes in MGs rely on concepts in which the physical system is modeled statically. For example, some of the developed methods to detect data integrity attacks fail to replicate dynamic systems such as MGs with different modes of operation [6], [7], [9]. Other approaches gather and process state data centrally [8], their computational efficiency is typically very low, and adverse attacks cannot be handled properly [12]. Furthermore, the aforementioned attack detection and identification approaches all require accurate mathematical models, which are almost impossible for most of cyber-physical systems. Even though the identification of attacks in a state space system has been studied beyond MGs in the literature, most of investigations require the system information [10], [17], [18]. For instance, in [20], the authors present an attack-resilient state estimation methodology that is able to detect attacks affecting the sensor measurements and reconstruct the state estimates using system information in the IEEE 34 node distribution test feeder.

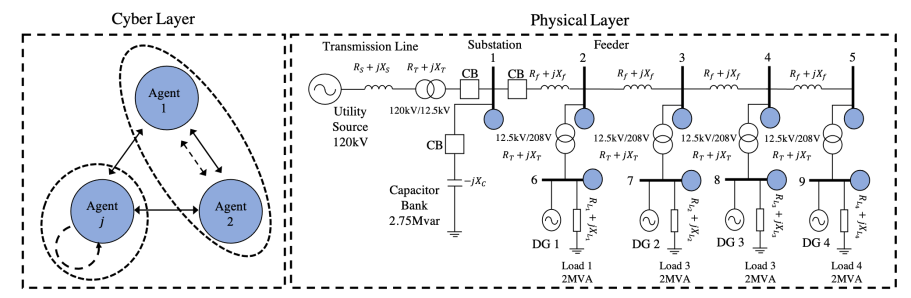

Fig. 1: Cyber-physical structure of four inverter-based DG MG system (Canadian urban benchmark distribution system).

Through the detection modeling framework of this work utilizing subspace identification, we show the (un)detectable conditions of the attack models (Theorem 2p. Existing methods from data-driven fault diagnosis literature do not consider threat models of stealthy attacks. Furthermore, such schemes rely on sparse recovery methods and cannot be extended to the identification of sparse attacks since the design of a bank of projection matrices or diagnostic observer is combinatorial complex [21]. Although our work focuses on effectively detecting malicious attacks, it leverages a threshold-based approach [20]. Contrary to [20], where corrupted system states could be recovered with 0.1 error, our proposed methodology is able to correctly identify corrupted measurements with a maximum error threshold of 0.06 .

\section{Cyber-Physical Structure of Islanded MG}

As any cyber-physical system, a multi-agent cyber-physical MG includes both a cyber and a physical layer. The physical system is the interconnected electric power system, while the cyber system is the communication network medium for data exchange between the physical agents of the MG. The structure of the cyber-physical MG used in this work is depicted in Fig. 11. The Canadian urban benchmark distribution system represents the physical layer of the inverter-based MG, while the cyber layer includes the connected agents placed in each bus of the distributed system architecture used in this case study [22]. For the modeling of the aforementioned physical layer of the distribution system, we have followed the mathematical description introduced by [13]. On top of the physical nodes, the cyber ICT layer links the various 


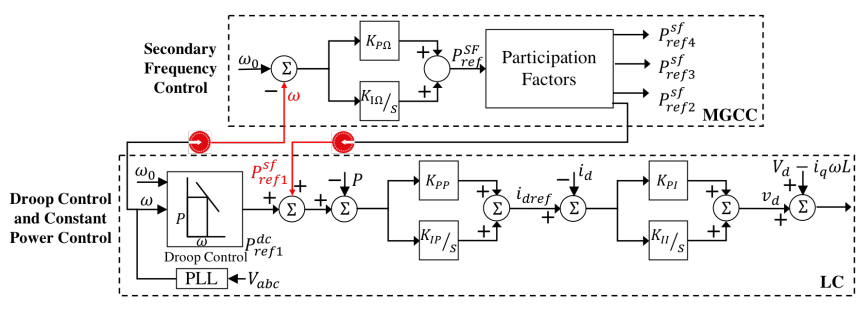

Fig. 2: Primary and secondary control of the multi-DG system.

cyber-physical agents. The physical dynamics of each agent are determined based on the states of other agents as well as its own state conditions via a centralized, distributed, or decentralized architecture. Such a structure is composed of $M$ cyber-physical multi-agent subsystems $\Omega_{j}, j \in \mathbb{N}: j \in[1, M]$ in which $y_{j}$ and $x_{j}$ are the measured output vector and system state of the $j_{t h}$-agent. $\tilde{\mathbf{x}}_{j}$ represents states of agents which are connected to agent $j$, and $\mathbf{x}_{j}, \forall j \in \mathbb{N}^{[1, M]}$ represents all system agents' state. The formulation of the $M$ agents' network is a directed graph $\mathcal{G}=(\mathcal{V}, \mathcal{E})$. The set of vertices/nodes (MG agents) is defined as $\mathcal{V}=\left\{v_{1}, \ldots, v_{M}\right\}$, and the set of edges as $\mathcal{E} \subseteq \mathcal{V} \times \mathcal{V}$ [23].

The inverter-based MG used for this work can be operated in either an autonomous or a grid-connected mode [22]. The inverter-based DGs can encompass solar PV modules, microturbines, fuel cells, etc. The MG can be considered as a system with $n$ buses and $n$ DGs. Buses without DGs can be abstracted to DG supported buses with zero generation capacity. Additionally, the MG has $n$ loads; similarly, no load buses can be seen as buses operating under zero load conditions. The benchmark parameters can be found in [13]. The hierarchical inverter control structure follows the literature in [13], [19], [24], with (i) local controllers (LCs) (primary) for the inverter frequency of the DGs and the distributed storage, and (ii) a MG centralized controller (MGCC) (secondary) at the low-voltage side of the substation [25].

The primary control operates independently, i.e., locally, within each corresponding DG. Local control exhibits fast response characteristics due to the negligible inertia and limited communication (e.g., when operating in autonomous mode). Thus, LCs are able to address load variations or arbitrary disturbances and effectively stabilize frequency dynamics. After frequency restoration, the secondary control can provide via the cyber layer auxiliary real power setpoints for LCs. The two-level control of every inverter-based DG for the MG is presented in Fig. 2. For the nominal operation of a DGbased MG, information is constantly exchanged between the DGs and the MGCC (highlighted in Fig. 2). However, LCs are sensitive to attacks targeting the control inputs or state measurements used for frequency and power regulation. Similarly, the secondary control mechanism can be compromised by adversaries using false measurement injections. In Section IV] we provide the mathematical formulation for such attack classes and demonstrate how they can destabilize the MG system under test (Section VI).

The small signal model of the autonomous MG is described based on its three blocks [26]: the inverter-based DG, the network and load block, and the interface block. Following the mathematical formulation presented in [13], the model of the islanded MG can be formulated as:

$$
\mathbf{\Phi} \Delta \dot{\mathbf{x}}=\mathbf{A} \Delta \mathbf{x}+\mathbf{F r}_{0}
$$

where $\mathbf{A}$ and $\mathbf{\Phi}$ are the system matrix and parameter matrix (singular), respectively. $\mathbf{x}$ and $\mathbf{r}_{0}$ are defined as: $\mathbf{r}_{0}=\left[\omega_{0}\right]^{T}$ and $\mathbf{x}=\left[\delta, \omega, \mathbf{i}_{\mathbf{d}}, \mathbf{i}_{\mathbf{q}}, \mathbf{i}_{\text {dref }}, \mathbf{i}_{\text {qref }}, \mathbf{v}_{\mathbf{d}}, \mathbf{v}_{\mathbf{q}}, \mathbf{P}, \mathbf{Q}, \mathbf{V}_{\mathbf{d}}, \mathbf{V}_{\mathbf{q}}, \mathbf{i}_{\mathbf{x}}, \mathbf{i}_{\mathbf{y}}\right.$, $\left.\mathbf{V}_{\mathbf{x}}, \mathbf{V}_{\mathbf{y}}\right]^{T}$, respectively. The expressions of the sparse matrices $\Phi$ and $\mathbf{A}$ can be found in Appendix A of [13].

By defining the state variable $\tilde{\mathrm{x}}$ shown in Eq. (2), the system's state space model reformulates as in Eq. (3).

$$
\begin{gathered}
\tilde{\mathbf{x}}=\left[\Delta \delta, \Delta \omega, \Delta \mathbf{i}_{\mathbf{d}}, \Delta \mathbf{i}_{\mathbf{q}}, \Delta \mathbf{i}_{\text {dref }}, \Delta \mathbf{i}_{\mathbf{q r e f}}, \Delta \mathbf{u}_{\mathbf{d}}, \Delta \mathbf{u}_{\mathbf{q}}\right]^{T} \\
\Delta \dot{\tilde{\mathbf{x}}}=\tilde{\mathbf{A}} \Delta \tilde{\mathbf{x}}
\end{gathered}
$$

where $\tilde{\mathbf{x}}=\left[\tilde{\mathbf{x}}_{1}, \ldots, \tilde{\mathbf{x}}_{m}\right]^{T}$ and:

$$
\begin{gathered}
\tilde{\mathbf{A}}=\left(\boldsymbol{\Phi}_{1}-\boldsymbol{\Phi}_{2} \mathbf{A}_{4}^{-1} \mathbf{A}_{3}\right)^{-1}\left(\mathbf{A}_{1}-\mathbf{A}_{2} \mathbf{A}_{4}^{-1} \mathbf{A}_{3}\right) \\
\boldsymbol{\Phi}=\left[\begin{array}{cc}
\boldsymbol{\Phi}_{1} & \boldsymbol{\Phi}_{2} \\
0 & 0
\end{array}\right] \text { and } \mathbf{A}=\left[\begin{array}{ll}
\mathbf{A}_{1} & \mathbf{A}_{2} \\
\mathbf{A}_{3} & \mathbf{A}_{4}
\end{array}\right]
\end{gathered}
$$

Based on the secondary frequency control, and defining the control output $u_{j}$ equal to the $P_{r e f_{j}}^{s f}$, i.e., the $\mathrm{DG} j_{\text {th }}$ auxiliary real power setpoint set by MGCC secondary control, and $y_{j}=$ $\omega_{j}$, the model of MG's output feedback controller is:

$$
\left\{\begin{array}{l}
\mathbf{u}=\mathbf{K y} \\
\mathbf{y}=\mathbf{C} \Delta \tilde{\mathbf{x}}
\end{array}\right.
$$

with $\mathbf{u}=\left[u_{1}, \ldots, u_{n}\right]^{T}, \mathbf{y}=\left[y_{1}, \ldots, y_{n}\right]^{T}$, and $\mathbf{C} \in \mathbb{R}^{n \times m}$ being the output matrix. As a result, the system model is:

$$
\left\{\begin{array}{l}
\Delta \dot{\tilde{\mathbf{x}}}=\tilde{\mathbf{A}} \Delta \tilde{\mathbf{x}}+\mathbf{B u} \\
\mathbf{y}=\mathbf{C} \Delta \tilde{\mathbf{x}}
\end{array}\right.
$$

where $\mathbf{u}, \mathbf{y}, \Delta \tilde{\mathbf{x}}$ essentially can be seen as the state space model inputs, measurement outputs, and states, respectively, of the system with cardinality $\mathcal{S}$, where $m=2 S-1$ and $n \geq m$ to enable for $\Delta \tilde{\mathbf{x}}$ at each DG $j \in \mathcal{S}$ to be determined.

For a more realistic characterization of the system model, process and measurement noises need to be included due to modeling approximations, model integration errors, and uncertainty. A commonly used assumption is that measurement and state disturbances are additive, independent of $\mathbf{u}$ and the initial states $\Delta \tilde{\mathbf{x}}(\mathbf{0})$, and normally distributed with zero mean and variance $\sigma_{j}^{2}$, i.e., $\mathbf{w} \sim \mathcal{N}\left(\mathbf{0}, \operatorname{diag}\left(\sigma_{1_{w}}^{2}, \ldots, \sigma_{n_{w}}^{2}\right)\right)$ and $\mathbf{v} \sim \mathcal{N}\left(\mathbf{0}, \operatorname{diag}\left(\sigma_{1_{v}}^{2}, \ldots, \sigma_{n_{v}}^{2}\right)\right)$ are the associated process and measurement noise, respectively [27].

$$
\left\{\begin{array}{l}
\Delta \dot{\tilde{\mathbf{x}}}=\tilde{\mathbf{A}} \Delta \tilde{\mathbf{x}}+\mathbf{B u}+\mathbf{w} \\
\mathbf{y}=\mathbf{C} \Delta \tilde{\mathbf{x}}+\mathbf{v}
\end{array}\right.
$$

Current approaches in detecting data integrity attacks within the communication of MGCC and LCs in such state space modeling involve using residual-based methods [28], [29]. The residual $\mathbf{r}$ is often determined from the difference of $\mathbf{y}$ and estimated values $\Delta \tilde{\mathbf{x}}$ for these data and the known topology matrix of the system C. As an example, a $\chi^{2}$-distribution with $n-m$ degrees of freedom and confidence interval can determine the threshold $\eta$ equal to $\sigma \sqrt{ } \chi_{n-m, \alpha}^{2}$. In the scenario of $\mathbf{r}=\|\mathbf{y}-\mathbf{C} \Delta \tilde{\mathbf{x}}\|_{2}>\eta$, then bad data will be detected. 


\section{ATtack Model Formulation}

Existing literature considering sparse false data assumes that adversaries have full knowledge of the power system configuration [14], [30]. Specifically, an attacker is considered to have infinite resources and knowledge with respect to: $(i)$ system topology including the status of switches and breakers, (ii) power lines limits and parameters, and (iii) ability to access the metering infrastructure and compromise sensing measurements. As a result, adversaries can estimate $\Delta \dot{\tilde{\mathbf{x}}}$ and construct $\mathbf{r}$ and $\eta$ in a way to not be detected. They can select a linear combination of the - known to them - Jacobian $\mathbf{C}$, i.e., $\lambda \mathbf{C}$, where $\lambda \in \mathbb{R}^{m \times 1}$. Given the attacked measurements $\mathbf{y}_{\alpha}$ on a subset of sensors or actuators $D_{\mathcal{S}}, \Delta \dot{\tilde{\mathbf{x}}}$ becomes $\Delta \dot{\tilde{\mathbf{x}}}_{\alpha} \neq \Delta \dot{\tilde{\mathbf{x}}}$, where $\alpha \in \mathcal{A}$ in the set of feasible attacks, and $\mathcal{A} \triangleq\left\{\alpha \in \mathbb{R}_{n}: \alpha_{j}=0, \forall j \notin D_{\mathcal{S}}\right\}$. In addition, due to the susceptibility of cyber-intrusions, existing works have considered both DoS as well as additive false data attacks on the feedback communication channels between MGCC and LCs [14], [19], [31]. Even though the attention of the majority of research is on attack detection and identification under sparse attacks [18], [32], most of them focus on additive attacks targeting the output vectors $\mathbf{y}$ [33].

In our attack model, the adversary follows a more realistic scenario without the requirement of full knowledge of the system topology, lines parameters, and component interconnection details. We adopt a 'blind' gray-box model in which the attacker can access the metering infrastructure and estimate a plausible attack vector based on historical measured data using blind source separation techniques [11], [34], [35]. The adversary has limited-knowledge in regards to the system's internals and with a partial understanding of the network and system model, which allows her/him to perform gray-box type of attacks. The limited adversary knowledge is realistic due to the restricted access to cyber-physical system control and monitoring functions, and errors in the data collection process. In this case, and under a fixed system topology described by $\mathbf{C}$, latent independent variables $\mathbf{z}_{L}$ (the loads of the system varying independently) and states $\mathbf{x}$ can be described as: $\mathbf{x}=f\left(\mathbf{C}, \mathbf{z}_{L}\right)$. State variables can be calculated using first-order Taylor series expansion $\mathbf{H}$ near $\mathbf{z}_{L}, \mathbf{x} \approx \mathbf{H z}_{L}$. Considering the state space model (Eq. (8)), the measurements can be now described in terms of load characterization as $\mathbf{y} \approx \mathbf{C H z}_{L}+\mathbf{v}$. If the adversary can access $\mathbf{C H}$, then an attack vector $\mathbf{y}_{\beta}$ can be formulated using a selected deviation in the flow of power $\delta \mathbf{z}_{L}$ such that $\mathbf{y}_{\beta}=\mathbf{y}+\mathbf{C H} \delta \mathbf{z}_{L}$. Generalizing the blind separation approach, $\mathbf{a}=\mathbf{G b}$, using a signal vector $\mathbf{b}$, an observed vector $\mathbf{a}$, and a fixed vector $\mathbf{G}$ as the mixing matrix, then the state model can be described in an equivalent manner as $\mathbf{y}=\mathbf{C H z}_{L}=\mathbf{G z}_{\mathbf{L}}$. If noises are of normal distribution without gross errors, $\mathbf{G}_{\mathbf{I C A}}=\mathbf{C H}$ can be determined using independent component analysis (ICA) [34], [35].

Our proposed attack model considers a blind attack in three different scenarios attacking actuators and sensors of the cyber-physical MG. The scenarios can be seen as 'black swan' events, i.e., highly unpredictable and improbable events that happen rarely and can potentially cause severe consequences.

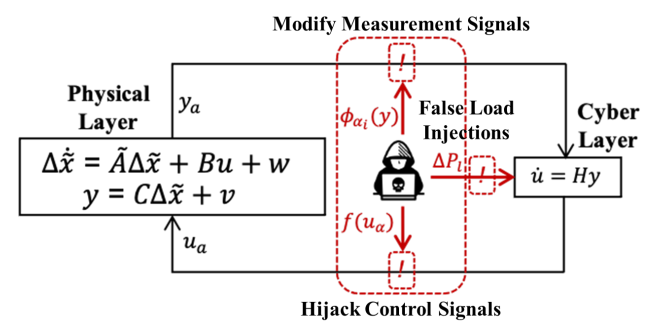

Fig. 3: The different types of cyberattacks in our model: (A) polluting the measurement vector, (B) hijacking input signals, and $(\mathrm{C})$ distorting the profile of loads.

Specifically, we adopt a stochastic monolithic system model which can capture the following formulations: (A) attack on measurement vector $\mathbf{y}$ by corrupting sparse measurements via data injection (Eq. (9)), (B) attack on the model input $\mathbf{u}$ by corrupting the integrity of inputs via sparse additive attacks (Eq. (10) ), and (C) a load deviation attack represented using the term $\Delta P_{l}$ [36] (Eq. (11). Fig. 3 depicts a diagram of the mathematical formulation and the respective variables compromised by attackers during the aforementioned attack scenarios. Each scenario is defined leveraging the mathematical formulation introduced in Eq. (8) to describe the dynamic state space model of a cyber-physical MG plant. The attacks are performed at the cyber-layer of the MG, which, it can be generally expressed as: $\dot{\mathbf{u}}=\mathbf{H y}$, where $\mathbf{H} \in \mathbb{R}^{l \times m}$ represents the control matrix [37]. First, the attackers can affect measurement signals $(\mathbf{y})$ via modification or fabrication (Eq. (9)). Second, the attackers can hijack model input controls (u) via interruption, e.g., delaying their acquisition or utilization by the system (Eq. [10)). Third, attackers can launch injections to the local loads in the form of load-altering attacks (Eq. (11)) which can lead to high operational costs (at the grid side), unsafe frequency excursions, and even severe frequency and voltage stability issues that can further instigate DG trips and cascading failures [38], [39].

$$
\operatorname{Model}(\mathrm{A}):\left\{\begin{array}{l}
\Delta \dot{\tilde{\mathbf{x}}}_{a}=\tilde{\mathbf{A}} \Delta \tilde{\mathbf{x}}_{a}+\mathbf{B} \mathbf{u}_{a}+\mathbf{w} \\
\phi_{\alpha_{\mathbf{i}}}(\mathbf{y})=\mathbf{C}_{a} \Delta \tilde{\mathbf{x}}_{a}+\mathbf{v}_{a}
\end{array}\right.
$$

where the attack function on measurement $\mathbf{y}, \phi_{\alpha_{\mathbf{i}}}(\mathbf{y})$, follows Definitions 1 and 2 .

$$
\text { Model }(\mathrm{B}):\left\{\begin{array}{l}
\Delta \dot{\tilde{\mathbf{x}}}_{a}=\tilde{\mathbf{A}} \Delta \tilde{\mathbf{x}}_{a}+\mathbf{B} f\left(\mathbf{u}_{a}\right)+\mathbf{w} \\
\mathbf{y}_{a}=\mathbf{C} \Delta \tilde{\mathbf{x}}_{a}+\mathbf{v}
\end{array}\right.
$$

where the attack function on the model inputs $\mathbf{u}, f\left(\mathbf{u}_{a}\right)$ follows the additive attack Definition 3 .

$$
\text { Model }(\mathrm{C}):\left\{\begin{array}{l}
\Delta \dot{\tilde{\mathbf{x}}}_{a}=\tilde{\mathbf{A}} \Delta \tilde{\mathbf{x}}_{a}+\mathbf{B} \mathbf{u}_{a}+\mathbf{\Delta} \mathbf{P}_{\mathbf{l}}+\mathbf{w} \\
\mathbf{y}_{a}=\mathbf{C} \Delta \tilde{\mathbf{x}}_{a}+\mathbf{v}
\end{array}\right.
$$

where the load deviation attack is captured by the term $\Delta P_{l}$, and adds nonlinear characteristics to the model [39].

The definitions for the attacks on $\mathbf{y}$ and $\mathbf{u}$ are given below.

Definition 1 (Sparse Attack). An attack vector $\alpha$ is $(\nu, n)$ sparse iffor $D_{\mathcal{S}} \subset \mathcal{S},\left\|\alpha_{j}\right\|=0$ for $\forall j \in D_{\mathcal{S}}{ }^{c}$, and $\left|D_{\mathcal{S}}\right| \leq \nu$. The set of malicious measurements is defined $\mathbb{C} \triangleq\left\{D_{\mathcal{S}}\right.$ : 


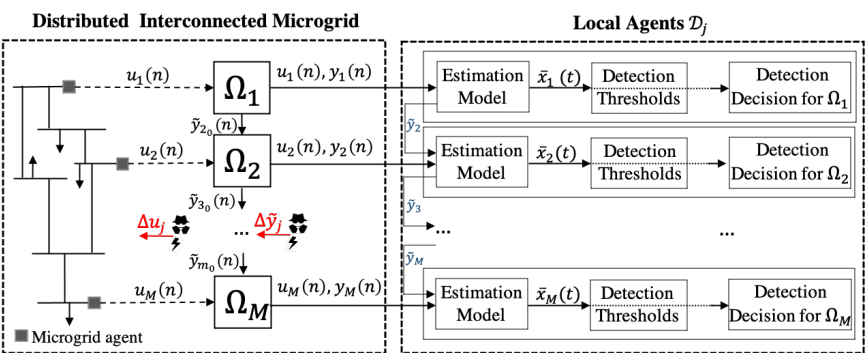

Fig. 4: Graphical depiction of the detection framework.

$\left.D_{\mathcal{S}} \subset \mathcal{S},\left|D_{\mathcal{S}}\right|=\nu\right\}$, while the set of all $(\nu, n)$-sparse attacks is represented as $\mathcal{A} \triangleq \bigcup_{D_{\mathcal{S}} \in \mathrm{C}}\left\{\alpha:\left\|\alpha_{j}\right\|=0, j \in D_{\mathcal{S}}{ }^{c}\right\}$.

Definition 2 (Data Injection Attack). An attack with a function $\phi_{\alpha_{i}}$ is called a data integrity or injection attack if a set of measurements are injected with false data. If $k_{1}$ denotes the beginning and $k_{2}$ the ending of the attack in discrete-time, and $\rho$ and $s$ are the received and sent signals, respectively, then:

$$
\rho[k]=\phi_{\alpha_{i}}(s[k])= \begin{cases}\alpha_{i}[k], & \text { if } k_{1}<k<k_{2} \\ s[k], & \text { else }\end{cases}
$$

where $\alpha_{i}[k] \neq s[k]$ is the attack signal. If $\alpha_{i}[k]$ is a data shift version of the original signal, i.e., $s[k]+\alpha_{s}[k]$ for $k_{1}<k<$ $k_{2}$, then the attack can be seen as a data shift attack.

Definition 3 (Sparse Additive Input Attack). The model of the system (Eq. (8)) under sparse additive attacks to its inputs is:

$$
\left\{\begin{array}{l}
\Delta \dot{\tilde{\mathbf{x}}}=\tilde{\mathbf{A}} \Delta \tilde{\mathbf{x}}+\mathbf{B}(\mathbf{u}+\mathbf{e})+\mathbf{w} \\
\mathbf{y}_{e}=\mathbf{C} \Delta \tilde{\mathbf{x}}+\mathbf{v}
\end{array}\right.
$$

where $\mathbf{e} \in \mathbb{R}^{n}$ stands for the sparse additive attack, assumed to be deterministic, with $e_{j}$ being zero or nonzero if the $j_{t h}$ sensor/actuator is attack-free or attacked, respectively. Let $K=\left\{i_{1}, \cdots, i_{|K|}\right\} \subset\left\{1, \cdots, k_{u}\right\}$ denote the attacked set. $|K|<k_{u}$ is the number of attacked sensors/actuators: all $K$-indexed items of $\mathbf{e}=\mathbf{e}(k)$ are nonzero at $k$.

\section{Detection Modeling Framework}

The framework achieves detection for distributed inverterbased DG clusters protecting against the attacks of Section IV The main concept is shown in Fig. 4. in which the distributed clusters are supported by local detection schemes $\mathcal{D}_{j}$ in the cyber-stage of the control layer [40], which allows more flexibility in containing cyberattack failures and preferred in recent times [41]. Each $\mathcal{D}_{j}$ is communicating with a subset of interconnected agents $\left[\mathcal{D}_{i}\right]$. During real-time operation, each agent utilizes adaptive detection rates, and the estimation models of subsystems dynamics. The decision outcome of each $\mathcal{D}_{j}$ is transferred to a high-level scheme, not to necessarily identify the event origin but to rather intelligently enable trustworthy attack detection. After detection, the risk can be mitigated via defensive and controlled isolation, by disconnecting vulnerable DG units and preventing propagation of attack impacts.

At the distribution grid level, we can represent each of the $M$ agents as $\Omega_{j}, j \in[1, M] \in \mathbb{K}$, described with a monolithic model capturing attack-free and attack models $(\mathrm{A}, \mathrm{B}, \mathrm{C})$ : $\hat{\mathbf{x}}(k)=f(\mathbf{x}(k), \mathbf{u}(k))+\beta(\mathbf{x}(k), \mathbf{u}(k))+\delta(\mathbf{x}(k), \mathbf{u}(k), k)$, where $\mathbf{x} \in \mathbb{R}^{m^{x}}$ and $\mathbf{u} \in \mathbb{R}^{n^{u}} .\{f, \beta, \delta\}: \mathbb{R}^{m^{x}} \times \mathbb{R}^{n^{u}}$ represents the system dynamics under nominal conditions, the adverse malicious effects including measurement noise and uncertainties, and the impact on system dynamics due to the divergence from the true model, respectively. Each of the $M$ subsystems can be then generically defined as:

$$
\Omega_{j}(\hat{\mathbf{x}} ; \mathbf{y}):\left\{\begin{aligned}
\hat{\mathbf{x}}_{j}(k) & =f\left(\mathbf{x}_{j}(k), \tilde{\mathbf{x}}_{j}(k), \mathbf{u}_{j}(k)\right) \\
& +\beta_{j}\left(\mathbf{x}_{j}(k), \tilde{\mathbf{x}}_{j}(k), \mathbf{u}_{j}(k)\right) \\
& +p_{j}^{x}\left(k-k_{0}^{x}\right) \delta_{j}\left(\mathbf{x}(k), \mathbf{u}_{j}(k)\right) \\
\mathbf{y}_{j}(k) & =C_{j} \mathbf{x}(k)+\zeta_{j}(k) \\
& +p_{j}^{y}\left(k-k_{0}^{y}\right) \sigma_{j}(k)
\end{aligned}\right.
$$

where $\mathbf{x}_{j} \in\left\{\Psi_{\mathbf{x}_{j}} \subset \mathbb{R}^{m^{x_{j}}}\right\}, \mathbf{u}_{j} \in\left\{\Psi_{\mathbf{u}_{j}} \subset \mathbb{R}^{n^{u_{j}}}\right\}, \mathbf{y}_{j} \in$ $\left\{\Psi_{\mathbf{y}_{j}} \subset \mathbb{R}^{n^{y_{j}}}\right\}$ are the state variables, the input vectors, and the measured output vectors of $\Omega_{j}$, respectively, and $\hat{\mathbf{x}}_{j}(k) \triangleq$ $\mathbf{x}_{j}(k+1)$ is the state of $\Omega_{j}$ at the $k+1$ time instant. $\tilde{\mathbf{x}}_{j}$ represents the states of the neighboring subsystems $\left[\Omega_{i}\right]$ that affect $\Omega_{j}$ (if $\tilde{\mathbf{x}}_{j}$ is dependent on a number of outputs of $\Omega_{i}, \mathbf{y}_{i}$ ). $p_{j}^{x}\left(k-k_{0}^{x}\right) \delta_{j}\left(\mathbf{x}, \mathbf{u}_{j}\right)$ denotes the dynamics due to malicious events impacting $\Omega_{j}$ and $p_{j}^{x}\left(k-k_{0}^{x}\right): \mathbb{R} \mapsto \mathbb{R}^{+}$is the time profile of an incipient or abrupt adverse event that occurs at some unknown time $n_{0}^{x}$. Note that $\delta_{j}$ may be determined using the global state $\mathbf{x}$ and not using solely the $j^{\text {th }}$ local states. $C_{j} \in \mathbb{R}^{n^{y_{j}}} \times \mathbb{R}^{m^{x_{j}}}$ is the known output matrix of $\Omega_{j}$ under normal operations. Note that due to Eq. (6), the models of Eq. (9) - 11) are captured sufficiently by Eq. (14). $\zeta_{j} \in\left\{\Psi_{\zeta_{j}} \subset\right.$ $\left.\mathbb{R}^{n^{y_{j}}}\right\}$ characterizes the measurement noise and $\sigma_{j}(k)$ denotes the attack model nature. Finally, $p_{j}^{y}\left(k-k_{0}^{y}\right) \sigma_{j}(k)$ characterizes the adverse event over its time evolution.

In order to calculate the attack detectable conditions of the proposed framework, first, we introduce the preliminary notions used throughout the rest of this Section:

Remark 1. Considering that some of $\tilde{\mathbf{x}}_{k}$ data are protected, we classify $\tilde{\mathbf{x}}_{j}, \forall j \in[1, M] \in \mathbb{K}^{m^{\tilde{\mathbf{x}}_{j}}}$ into four types: 1) unprotected due to at most a number $m$ of $\tilde{\mathbf{x}}_{k}$ for $k \in \Gamma$, $\Gamma \subset m^{\tilde{\mathbf{x}}}$ is the index set of such data and the cardinality of $\Gamma$ is $\operatorname{card}(\Gamma)=m$, 2) protected not subjected to incidental and intentional perturbation with index $\Gamma^{A}=m^{\tilde{\mathbf{x}}} / \Gamma$ and $\operatorname{card}\left(\Gamma^{A}\right)=M-m=\mu$, 3) corrupted $\tilde{\mathbf{x}}_{k_{\alpha}}$ for $k \in \Gamma^{\alpha}$ : $\Gamma^{\alpha} \subset \Gamma, \tilde{\mathbf{x}}_{k_{\alpha}} \neq \tilde{\mathbf{x}}_{k_{0}} \forall k \in \Gamma^{\alpha}$, and $\operatorname{card}\left(\Gamma^{\alpha}\right)$ is $0 \leq \Gamma^{\alpha} \leq m$, 4) healthy for which $\Gamma^{\mu}=m^{\tilde{\mathbf{x}}} / \Gamma^{\alpha}$ and $\tilde{\mathbf{x}}_{k}=\tilde{\mathbf{x}}_{k_{0}} \forall k \in \Gamma^{\mu}$.

Remark 2. For each $\Omega_{j}$ in Eq. (14) which $f\left(\mathbf{x}_{j}, \tilde{\mathbf{x}}_{j}, \mathbf{u}_{j}\right)=$ $A_{j} \mathbf{x}_{j}$, the pair $\left(A_{j}, C_{j}\right)$ is considered detectable.

Remark 3. For every $\Omega_{j}$ described in Eq. (14), $\mathbf{x}_{j}, \mathbf{u}_{j}$, and $\zeta_{j}$ belong to a known compact region $\Psi_{\mathbf{x}_{j}}, \Psi_{\mathbf{u}_{j}}$, and $\Psi_{\zeta_{j}}$, respectively. This allows for a well-posedness problem while differentiating between noise and malicious events.

Remark 4. The modeling uncertainty $\eta_{j}$ of the adverse effect $\beta_{j}(k)$ of nature $\sigma_{j}(k)$ is considered unknown and unstructured, but bounded under "golden" (attack-free) conditions. i.e., $\left\|\eta_{j}\left(\mathbf{x}_{j}, \tilde{\mathbf{x}}_{j}, \mathbf{u}_{j}, k\right)\right\| \leq\left\|\tilde{\eta}_{j}\left(\mathbf{y}_{j}, \tilde{\mathbf{y}}_{j}, \mathbf{u}_{j}\right)\right\|, \forall k \in \mathbb{K}$, $\forall\left(\mathbf{x}_{j}, \tilde{\mathbf{x}}_{j}, \mathbf{u}_{j}\right) \in \Psi_{j}$. We assume that the noisy counterpart of $\tilde{\mathbf{y}}_{j_{0}}(k)$ is given by $\tilde{\mathbf{y}}_{j}(k)=\tilde{\mathbf{y}}_{j_{0}}(k)+\zeta_{j}$, and $\tilde{\eta}_{j}\left(\mathbf{y}_{j}, \tilde{\mathbf{y}}_{j}, \mathbf{u}_{j}\right) \geq 0$ represents a known function in the region of interest $\Psi_{j}=$ 
$\Psi_{\mathbf{x}_{j}} \times \Psi_{\tilde{\mathbf{x}_{j}}} \times \Psi_{\mathbf{u}_{j}} \subset \mathbb{R}^{m^{x_{j}}} \times \mathbb{R}^{m^{\tilde{x_{j}}}} \times \mathbb{R}^{n^{u_{j}}}$. The bound $\tilde{\eta}_{j}$ enables distinguishable attack events from modeling uncertainty.

For simplicity, in Fig. 4. $\tilde{\mathbf{y}}_{j}(k)$ is defined as $C_{j} \tilde{\mathbf{x}}_{j}(k)$ and $\tilde{\mathbf{y}}_{j_{0}}(k)$ represents the "golden" (noiseless and attackfree) version of $\tilde{\mathbf{y}}_{j}(k)$. The detectability of attacks is based on data-driven subspace methods, wherein the detector is constructed via identifying the stable kernel representation (SKR) in the attack-free case. Our local estimation models for each $\Omega_{j}$ towards detecting the attacks, and following Eq. (14), consider an observation model in Eq. (15) under attack-free conditions, where $G_{j}$ is a gain matrix of the measured and estimated outputs to be determined such that $\left(A_{j}-G_{j} C_{j}\right)$ is Schur stable. If the pair $\left(A_{j}, C_{j}\right)$ is considered detectable, i.e., $f\left(\mathbf{x}_{j}, \tilde{\mathbf{x}}_{j}, \mathbf{u}_{j}\right)=A_{j} \mathbf{x}_{j}$, (Remark 2), $G_{j}$ can always be determined. In case the attack-free condition is violated, Eq. 15 includes the measurement error $\epsilon_{j} \in \mathbb{R}^{n^{y_{j}}}$ attributed to noise or/and the occurrence of an attack event, i.e., $\overline{\mathbf{y}}_{j}(k)=C_{j} \overline{\mathbf{x}}_{j}(k)+\epsilon_{j}$. In this case, the local model will result in erroneous system inference.

$$
\left\{\begin{aligned}
\overline{\mathbf{x}}_{j}(k) & =A_{j} \mathbf{x}_{j}(k)+\beta_{j}\left(\mathbf{y}_{j}(k), \tilde{\mathbf{y}}_{j}(k), \mathbf{u}_{j}(k)\right) \\
& +G_{j}\left(\mathbf{y}(k)-\overline{\mathbf{y}}_{j}(k)\right) \\
\overline{\mathbf{y}}_{j}(k) & =C_{j} \overline{\mathbf{x}}_{j}(k)
\end{aligned}\right.
$$

Following the inverter-based MG model of Eq. 8) and the framework modeling of the distributed inverter-based DG clusters of Eq. (14), the attack-free sequence model of Eq. (15) if we collect $q>m$ measurements starting from $k$ is:

$$
\mathbf{y}_{q}(k)=\boldsymbol{\Gamma}_{q} \mathbf{x}(k)+\mathbf{H}_{u, q} \mathbf{u}_{q}(k)+\mathbf{H}_{w, q} \mathbf{w}_{q}(k)+\mathbf{v}_{q}(k)
$$

where

$$
\mathbf{y}_{q}(k)=[y(k), y(k+1), \cdots, y(k+q-1)]^{T} \in \mathbb{R}^{q n}
$$

represents the sequence from $[k]$ to $[k+q-1]$ of $\mathbf{y}(k)$, and $q \in \mathbb{Z}^{+}$denotes the length of the sequence, and

$$
\boldsymbol{\Gamma}_{q}=\left[\mathbf{C}, \mathbf{C A}, \cdots, \mathbf{C A}^{q-1}\right]^{T} \in \mathbb{R}^{q n \times m}
$$

denotes the extended observability matrix, and

$$
\begin{aligned}
\mathbf{H}_{u, q}= & {\left[\begin{array}{cccc}
\mathbf{I} & 0 & \cdots & 0 \\
\mathbf{C B} & \mathbf{I} & \cdots & 0 \\
\vdots & \ddots & \ddots & \vdots \\
\mathbf{C A}^{q-2} \mathbf{B} & \cdots & \mathbf{C B} & \mathbf{I}
\end{array}\right] \in \mathbb{R}^{q n \times q n} } \\
\mathbf{H}_{w, q} & =\left[\begin{array}{cccc}
0 & 0 & \cdots & 0 \\
\mathbf{C} & 0 & \cdots & 0 \\
\vdots & \ddots & \ddots & \vdots \\
\mathbf{C A}^{q-2} & \cdots & \mathbf{C} & 0
\end{array}\right] \in \mathbb{R}^{q n \times q m}
\end{aligned}
$$

represent the I/O and process noise-output Toeplitz matrices.

Taking into account the attack models $(\mathrm{A}, \mathrm{B}, \mathrm{C})$ formalized in our framework representation of Eq. (14), the sequence model of the MG under attacks of nature $\sigma_{j}(k)$ can be described as:

$$
\begin{aligned}
\mathbf{y}_{q}(k)= & \boldsymbol{\Gamma}_{q} \mathbf{x}(k)+\mathbf{H}_{u, q} \mathbf{u}_{q}(k)+\mathbf{H}_{u, q} \boldsymbol{\Sigma}_{e}(k) \mathbf{u}_{q}(k) \\
& +\mathbf{H}_{w, q} \mathbf{w}_{q}(k)+\mathbf{v}_{q}(k)
\end{aligned}
$$

where $\boldsymbol{\Sigma}_{e}(k)$ is the attack related matrix of attack models $\sigma_{j}(k)$ over their time evolution $p_{j}^{x}(k+q-1)$ :

$$
\boldsymbol{\Sigma}_{e}(k)=\left[\begin{array}{ccc}
\sigma(k) & \cdots & 0 \\
\vdots & \ddots & \vdots \\
0 & \cdots & \sigma(k+q-1)
\end{array}\right] \in \mathbb{R}^{q n \times q n}
$$

For an oblivious adversary, following a 'blind' attack model without full knowledge of system topology, lines parameters, and components interconnections, neither the time-invariant system model of Eqs. (8) and (14) nor the sequence model of Eq. (20) can be directly used for securing tight attack detection thresholds as the system matrices $A, B, C$ are unknown. To realize the efficient system identification, we place the available data in a matrix resulting in the corresponding data model:

$$
\mathbf{Y}_{k, q}=\boldsymbol{\Gamma}_{q} \mathbf{X}_{k}+\mathbf{H}_{u, q} \mathbf{U}_{k, q}+\mathbf{H}_{w, q} \mathbf{W}_{k, q}+\mathbf{V}_{k, q}
$$

where $\mathbf{Y}_{k, q} \in \mathbb{R}^{q n \times N}, \mathbf{X}_{k} \in \mathbb{R}^{m \times N}, \mathbf{U}_{k, q} \in \mathbb{R}^{q n \times N}, \mathbf{W}_{k, q} \in$ $\mathbb{R}^{q m \times N}, \mathbf{V}_{k, q} \in \mathbb{R}^{q n \times N}$ are defined based on the notation:

$$
\boldsymbol{\Psi}_{k, q}=\left[\boldsymbol{\Psi}_{q}(k) \cdots \boldsymbol{\Psi}_{q}(k+N-1)\right] \in \mathbb{R}^{q k_{\phi} \times N}
$$

where $\boldsymbol{\Psi}_{k} \in \mathbb{R}^{k_{\psi} \times N}$ in form of Eq. (17) and $N \in \mathbb{Z}, N \gg m$.

Considering the models of Eqs. 16) and 20, the sequence model in both the noise- and attack- free case becomes:

$$
\mathbf{y}_{q}(k)=\boldsymbol{\Gamma}_{q} \mathbf{x}(k)+\mathbf{H}_{u, q} \mathbf{u}_{q}(k)
$$

Let $\boldsymbol{\Gamma}_{q}^{\perp}$ satisfy $\boldsymbol{\Gamma}_{q}^{\perp} \boldsymbol{\Gamma}_{q}=0$, if we multiply $\boldsymbol{\Gamma}_{q}^{\perp}$ on Eq. 24]:

$$
\boldsymbol{\Gamma}_{q}^{\perp} \mathbf{y}_{q}(k)=\boldsymbol{\Gamma}_{q}^{\perp} \mathbf{H}_{u, q} \mathbf{u}_{q}(k)
$$

Based on Eq. 25], the detector of DG subsystems is defined:

$$
\mathbf{r}(k)=\left[-\boldsymbol{\Gamma}_{q}^{\perp} \mathbf{H}_{u, q} \quad \boldsymbol{\Gamma}_{q}^{\perp}\right]\left[\begin{array}{c}
\mathbf{u}_{q}(k) \\
\mathbf{y}_{q}(k)
\end{array}\right]
$$

in which $\mathbf{r}(k)=0$ represents the noise- and attack- free case. In the next part, we demonstrate the realization of the attack detector in Eq. (26) using subspace identification methods.

\section{A. Attack Detection}

Before we construct the attack detectable conditions, we provide the preliminaries for SKR and its subspace identification.

Definition 4 (Data-driven SKR). For the model of Eqs. (8) and (14) and with $\mathbf{w}(k)$ and $\mathbf{v}(k)$ being zero, the matrix $\mathcal{K}_{d, q}$ is the data-based realization of $S K R$, if for $q \in \mathbb{Z}$ it holds:

$$
\mathcal{K}_{d, q}\left[\begin{array}{l}
\mathbf{u}_{q}(k) \\
\mathbf{y}_{q}(k)
\end{array}\right]=0, \forall \mathbf{u}_{q}(k), \mathbf{x}(0)
$$

In the noise-free case, $\mathcal{K}_{d, q}$ is located in the left null subspace of the image subspace determined by the inputs and outputs of Eq. (8). Due to the requirement of identifying the image subspace, we rewrite the data model of Eq. 22) as:

$$
\left[\begin{array}{c}
\mathbf{U}_{k, q} \\
\mathbf{Y}_{k, q}
\end{array}\right]=\left[\begin{array}{cc}
\mathbf{I} & \mathbf{0} \\
\mathbf{H}_{u, q} & \boldsymbol{\Gamma}_{q}
\end{array}\right]\left[\begin{array}{c}
\mathbf{U}_{k, q} \\
\mathbf{X}_{k}
\end{array}\right]+\left[\begin{array}{c}
0 \\
\mathbf{H}_{w, q} \mathbf{W}_{k, q}+\mathbf{V}_{k, q}
\end{array}\right]
$$

where

$$
\left[\begin{array}{c}
\overline{\mathbf{U}}_{k, q} \\
\overline{\mathbf{Y}}_{k, q}
\end{array}\right]=\left[\begin{array}{cc}
\mathbf{I} & \mathbf{0} \\
\mathbf{H}_{u, q} & \boldsymbol{\Gamma}_{q}
\end{array}\right]\left[\begin{array}{c}
\mathbf{U}_{k, q} \\
\mathbf{X}_{k}
\end{array}\right]
$$


can be seen as the input and output data matrix in the noisefree scenario and obtained by subtracting $\mathbf{H}_{w, q} \mathbf{W}_{k, q}+\mathbf{V}_{k, q}$.

Let matrices $\mathbf{P}$ and $\mathbf{F}$ represent past and future data:

$$
\mathbf{P}=\left[\begin{array}{c}
\mathbf{U}_{k-p, p} \\
\mathbf{Y}_{k-p, p}
\end{array}\right], \quad \mathbf{F}=\left[\begin{array}{c}
\mathbf{U}_{k, q} \\
\mathbf{Y}_{k, q}
\end{array}\right]
$$

where the index $p$ is the past data sequence length. According to $L Q$ decomposition, we can introduce:

$$
\left[\begin{array}{c}
\mathbf{P} \\
\mathbf{U}_{k, q} \\
\mathbf{Y}_{k, q}
\end{array}\right]=\left[\begin{array}{ccc}
\mathbf{R}_{11} & 0 & 0 \\
\mathbf{R}_{21} & \mathbf{R}_{22} & 0 \\
\mathbf{R}_{31} & \mathbf{R}_{32} & \mathbf{R}_{33}
\end{array}\right]\left[\begin{array}{l}
\mathbf{Q}_{1} \\
\mathbf{Q}_{2} \\
\mathbf{Q}_{3}
\end{array}\right]
$$

Consequently, the future matrices $\mathbf{U}_{k, q}$ and $\mathbf{Y}_{k, q}$ satisfy:

$$
\left[\begin{array}{c}
\mathbf{U}_{k, q} \\
\mathbf{Y}_{k, q}
\end{array}\right]=\left[\begin{array}{ll}
\mathbf{R}_{21} & \mathbf{R}_{22} \\
\mathbf{R}_{31} & \mathbf{R}_{32}
\end{array}\right]\left[\begin{array}{l}
\mathbf{Q}_{1} \\
\mathbf{Q}_{2}
\end{array}\right]+\left[\begin{array}{c}
0 \\
\mathbf{R}_{33} \mathbf{Q}_{3}
\end{array}\right]
$$

Since $\mathbf{W}_{k, q}$ and $\mathbf{V}_{k, q}$ are independent of the future input data $\mathbf{U}_{k, q}$ and the past data $\mathbf{P}$, it is demonstrated that:

$$
\begin{aligned}
& \mathbf{H}_{w, q} \mathbf{W}_{k, q}+\mathbf{V}_{k, q}=\mathbf{R}_{33} \mathbf{Q}_{3} \\
& {\left[\begin{array}{l}
\overline{\mathbf{U}}_{k, q} \\
\overline{\mathbf{Y}}_{k, q}
\end{array}\right]=\left[\begin{array}{ll}
\mathbf{R}_{21} & \mathbf{R}_{22} \\
\mathbf{R}_{31} & \mathbf{R}_{32}
\end{array}\right]\left[\begin{array}{l}
\mathbf{Q}_{1} \\
\mathbf{Q}_{2}
\end{array}\right]}
\end{aligned}
$$

The knowledge of the I/O data matrix of Eq. (33b) can lead to the identification of $\mathcal{K}_{d, q}$ according to Lemma 1 .

Lemma 1. The data-driven realization of $S K R \mathcal{K}_{d, q}=\mathbf{U}_{2}^{T}$ can be constructed via singular value decomposition (SVD):

$$
\left[\begin{array}{ll}
\mathbf{R}_{21} & \mathbf{R}_{22} \\
\mathbf{R}_{31} & \mathbf{R}_{32}
\end{array}\right]=\left[\begin{array}{ll}
\mathbf{U}_{1} & \mathbf{U}_{2}
\end{array}\right]\left[\begin{array}{cc}
\mathbf{S}_{1} & 0 \\
0 & \mathbf{S}_{2}
\end{array}\right]\left[\begin{array}{ll}
\mathbf{V}_{1}^{T} & \mathbf{V}_{2}^{T}
\end{array}\right]
$$

where $\mathbf{S}_{2} \approx 0$.

Proof of Lemma 1] The realization of SKR $\mathcal{K}_{d, q}$ satisfies:

$$
\mathcal{K}_{d, q}\left[\begin{array}{c}
\overline{\mathbf{U}}_{k, q} \\
\mathbf{Y}_{k, q}
\end{array}\right]=0
$$

Based on Eq. 33b, Eq. 35 can be transformed into:

$$
\mathcal{K}_{d, q}\left[\begin{array}{ll}
\mathbf{R}_{21} & \mathbf{R}_{22} \\
\mathbf{R}_{31} & \mathbf{R}_{32}
\end{array}\right]=0
$$

Then, combining Eq. (36) with Eq. (34), one can obtain that:

$$
\mathcal{R}\left(\left[\begin{array}{ll}
\mathbf{R}_{21} & \mathbf{R}_{22} \\
\mathbf{R}_{31} & \mathbf{R}_{32}
\end{array}\right]\right) \models \mathcal{R}\left(\mathbf{U}_{1}\right)
$$

and $\mathcal{K}_{d, q}=\mathbf{U}_{2}^{T}$.

Following the SKR identification of $\mathcal{K}_{d, q}$, the attack detector of Eq. 26) could be realized in a similar fashion:

$$
\mathbf{r}(k)=\mathcal{K}_{d, q}\left[\begin{array}{l}
\mathbf{u}_{q}(k) \\
\mathbf{y}_{q}(k)
\end{array}\right]
$$

where parameter matrices $\boldsymbol{\Gamma}_{q}^{\perp} \mathbf{H}_{u, q}$ and $\boldsymbol{\Gamma}_{q}^{\perp}$ can be estimated as:

$$
\mathcal{K}_{d, q}=\left[\begin{array}{ll}
-\boldsymbol{\Gamma}_{q}^{\perp} \mathbf{H}_{u, q} & \boldsymbol{\Gamma}_{q}^{\perp}
\end{array}\right]
$$

and $\Gamma_{q}^{\perp}$ is computed by extracting the last $q n$ columns of $\mathcal{K}_{d, q}$.
Theorem 1 (Attack Detector). If the attack detector is developed according to Eq. (38) and the residual estimation function follows Eq. (40):

$$
\mathbf{J}=\mathbf{r}^{T}(k) \mathbf{S}_{r}^{-1} \mathbf{r}(k)
$$

where $\mathbf{S}_{r}$ is given by:

$$
\mathbf{S}_{r}=\frac{1}{N-1} \boldsymbol{\Gamma}_{q}^{\perp} \mathbf{R}_{33} \mathbf{Q}_{3}\left(\boldsymbol{\Gamma}_{q}^{\perp} \mathbf{R}_{33} \mathbf{Q}_{3}\right)^{T}
$$

then the threshold of each detection agent $\mathcal{D}_{j}$ can be designed by $d_{j}=\chi_{1-\alpha}^{2}\left(k_{r}\right)$ with $k_{r}=q n-n$, and the detection logic being: $d \leq d_{j}$ if attack-free, or $d>d_{j}$ if attacked.

Proof of Theorem 1] If the attack detector is constructed according to Eq. 38, then the attack-free residual satisfies:

$$
\mathbf{r}_{0}(k)=\boldsymbol{\Gamma}_{q}^{\perp}\left(\mathbf{H}_{w, q} \mathbf{w}_{q}(k)+\mathbf{v}_{q}(k)\right)
$$

Due to Eq. (33a), the covariance of residual $\mathbf{S}_{r}$ in the attackfree case can be estimated by Eq. (41). Let the residual function be $\mathbf{d}=\mathbf{r}^{T}(k) \mathbf{S}_{r}^{-1} \mathbf{r}(k)$. The dimension of the residual is $k_{r}$, thus $\mathbf{d} \sim \chi^{2}\left(k_{r}\right)$. Setting the false rate of detection as $\alpha$, then the threshold is selected as $d_{j}=\chi_{1-\alpha}^{2}\left(k_{r}\right)$, and the detection logic can be designed as Theorem 1 .

1) Attack Detectable Conditions: The residual dynamics of the MG model under the attack models of Eqs. (9) - 111) and represented in the model of Eq. (14) are as follows:

$$
\begin{aligned}
\mathbf{r}_{\alpha}(k) & =\boldsymbol{\Gamma}_{q}^{\perp}\left(\mathbf{H}_{w, q} \mathbf{w}_{q}(k)+\mathbf{v}_{q}(k)\right) \\
& +\boldsymbol{\Gamma}_{q}^{\perp} \mathbf{H}_{u, q} \boldsymbol{\Sigma}_{e}(k) \mathbf{u}_{q}(k)
\end{aligned}
$$

where $\mathbf{r}_{\alpha}(k)$ denotes the residual caused by the attack vectors. The following theorem illustrates the detectable condition by the detector of Eq. (38). The resulting synergy of the attack detectability property can enhance system visibility and reveal attacks of models (A,B,C) even when load subsystems are subject to malicious manipulation (e.g., demand-side attacks).

Theorem 2 (Attack Detectable Condition). Given the datadriven attack detection of Eq. (38), the attack related matrix $\boldsymbol{\Sigma}_{e}(k)$ of attack models $\sigma_{j}(k)$ is detectable if and only if:

$$
\boldsymbol{\Gamma}_{q}^{\perp} \mathbf{H}_{u, q} \boldsymbol{\Sigma}_{e}(k) \mathbf{u}_{q}(k) \neq 0
$$

Proof of Theorem 2. (If): For the sake of contradiction, assume that $\boldsymbol{\Sigma}_{e}(k)$ is undetectable. Since $\boldsymbol{\Sigma}_{e}(k)$ is deterministic and the noise information $\boldsymbol{\Gamma}_{q}^{\perp}\left(\mathbf{H}_{w, q} \mathbf{w}_{q}(k)+\mathbf{v}_{q}(k)\right)$ is stochastic, the only possibility to guarantee the equality $\mathbf{r}_{\alpha}^{T}(k) \boldsymbol{\Sigma}_{r}^{-1}(k) \mathbf{r}_{\alpha}(k)=\mathbf{r}_{0}^{T}(k) \boldsymbol{\Sigma}_{r}^{-1}(k) \mathbf{r}_{0}(k)$ is that $\mathbf{r}_{\alpha}(k)=$ $\mathbf{r}_{0}(k)$. As a result, $\boldsymbol{\Gamma}_{q}^{\perp} \mathbf{H}_{u, q} \boldsymbol{\Sigma}_{e}(k) \mathbf{u}_{q}(k)=\mathbf{r}_{\alpha}(k)-\mathbf{r}_{0}(k)=0$ which contracts Eq. (44).

(Only If): Again resort to contradiction, assume $\boldsymbol{\Sigma}_{e}(k)$ fails Eq. 44, then $\boldsymbol{\Gamma}_{q}^{\perp} \mathbf{H}_{u, q} \boldsymbol{\Sigma}_{e}(k) \mathbf{u}_{q}(k)=0$. It follows from Eq. (43) that $\mathbf{r}_{\alpha}(k)=\boldsymbol{\Gamma}_{q}^{\perp}\left(\mathbf{H}_{w, q} \mathbf{w}_{q}(k)+\mathbf{v}_{q}(k)\right)=\mathbf{r}_{0}(k)$. Hence, the statistic properties of $\mathbf{r}_{\alpha}(k)$ are consistent with $\mathbf{r}_{0}(k)$. In this case, $\boldsymbol{\Sigma}_{e}(k)$ is undetectable by the attack detector of Eq. (38), which contracts the assumption that $\boldsymbol{\Sigma}_{e}(k)$ is detectable. 


\section{Simulation Results}

In this section, we demonstrate the detection and impact on the MG operation of the blind data attacks of the three scenarios in Eq. (9) - (11) targeting the DGs. We first present the effect on the voltage, frequency, and power measurements in case of the occurrence of each individual attack class in the control of the inverter-based DGs composing the MG. Then, we compute the residual dynamics of the MG model under the most impactful type of attack and demonstrate the voltage and frequency deviations on the DGs during sequential attacks on the MG (of the same most impactful attack class). Finally, we present how the detectable conditions allow for potent attack detection and DG sectionalization, minimizing the impact on the MG and grid operation.

\section{A. Simulation Model}

The simulations are performed in MATLAB/Simulink Power System environment in which the Canadian urban distribution system model of Fig. 1 is implemented. In more detail, the utility source node is operating at $120 \mathrm{kV}$ and is connected to a $12.5 \mathrm{kV} / 10 \mathrm{MVA}$ transformer. The rest of the grid is interfaced to the substation transformer via a circuit breaker, while a $2.75 \mathrm{MVar}$ capacitor bank is also installed at the substation bus for frequency and harmonic compensation purposes. The four inverter-based DGs - operating at $208 \mathrm{~V}$ level - are distributed along the feeder and connected with the corresponding DG buses via $12.5 \mathrm{kV} / 208 \mathrm{~V}$ step-down transformers. The loads connected to each of the DG buses are assumed to have a power factor of 0.95 .

The DGs are responsible to meet their inherent power demand. The DG inverter controllers utilize: (i) a phaselocked loop subsystem to assist the automatic gain control and track the operating frequency, and (ii) a discrete proportionalintegral (PI) controller tracking the DG-generated peak voltage used to control the pulse-width-modulation input supplied to the inverter's gate drivers. Additionally, the DGs are equipped with islanding mechanisms which can defensively sectionalize them once abnormal frequency and/or voltage conditions are encountered.

\section{B. Small Signal and Transient Stability Preliminaries}

Power system disturbances refer to unexpected changes encountered in system states. The system states model the voltage, current, frequency and power parameters as well as the physical properties of the grid architecture (i.e., topology and asset placement). Severe disturbances include fault conditions, which are typically followed by generation losses (if faults occur on the transmission level), and load shedding events to maintain power system operation. The rapid changes in system states during such critical conditions require extensive modeling since linearized equations are incapable to capture the transient nature of such events. Thus, transient stability analyses are critical to describe the events taking place in such scenarios [42]-[44]. However, the complexity of such analyses and the computational resources required to model and simulate interconnected power systems creates modeling challenges. Although the aforementioned events can have significant impacts on system performance, they can also be promptly detected with remediation plans being in place to overcome such adverse events.

On the other hand, a sophisticated and persistent adversary aiming at maliciously modifying the system operation could avoid being identified from such disturbance-type scenarios. By incorporating such adversaries as part of our threat model, we investigate the autonomous operation of cyber-physical MGs under small-magnitude attacks targeting the measurement vectors (VI-C), the inverter control inputs (VI-D), and load demand (VI-E). As a result, such minor disturbances can be investigated using small signal stability analysis, avoiding the complex system dynamics modeling required for transient stability analyses [45], [46]. The nature and sophistication of such attacks renders them more difficult to detect and, in this section, we demonstrate how the proposed methodology can efficiently identify them, avoiding any potential disturbances on the MG connected assets.

\section{Attacks Targeting the Measurement Vectors}

In this attack model (Model (A) of Eq. (9)), the DG's realtime measurements are stealthily modified in an effort to cause the DG/MG operation to become unstable compromising the DG inherent dependencies (e.g., loads) and potentially harming other grid equipment (e.g., transformers). To illustrate the impact of such data tampering attacks, we simulate a scenario in which the DG voltage regulator controller is compromised. Specifically, the DG peak voltage sensor measurements, which serve as inputs to the DG regulator, are reduced by $50 \%$ for $20 \mu \mathrm{s}$, and then nominal measurements are supplied to the controller. As a result, the data integrity attack on the measurement vector, and thus the impact on the DG's voltage state leads to unregulated output voltage behavior which can be observed in Fig. 5b. Although the DG frequency is minimally affected, both the DG's generated active and injected reactive power are severely impacted when compared to the nominal operating conditions (Fig.5a). Evidently, despite the short timing window of the attack, if the DGs are not promptly isolated from the MG, the voltage magnitude, active and reactive power fluctuations could jeopardize the system operation.

\section{Attacks Targeting the Control Inputs}

In this model (Model (B) of Eq. (10)), the DG control input is attacked via a sparse additive attack model aiming to cause voltage, frequency, and power fluctuations, which could further result in suboptimal power dispatch and uneconomic grid operation, and even require defensive islanding leading to loss of power or equipment damages. To exhibit the severity of this attack, we simulate a corresponding scenario in which the DG inverter's modulation control inputs are being tampered. In particular, we introduce an additive attack $\mathbf{e}=\mathbf{e}(k)$ on the $k$ inverter's input by superimposing a 'small' sinusoidal signal with random amplitude, i.e., $|\mathbf{e}| \in[0,0.1]$. The results of the attack on the model input are presented in Fig. 5c. We observe that the DG controller is able to retain stability due to the small control attack signal e. In case of larger control perturbations, the DG operation significantly deteriorates. Moreover, even in 

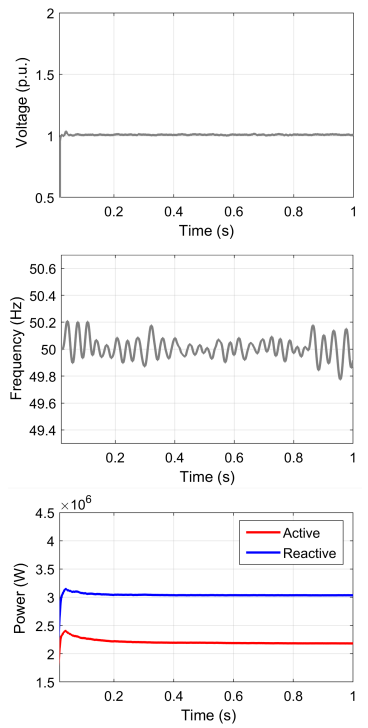

(a)
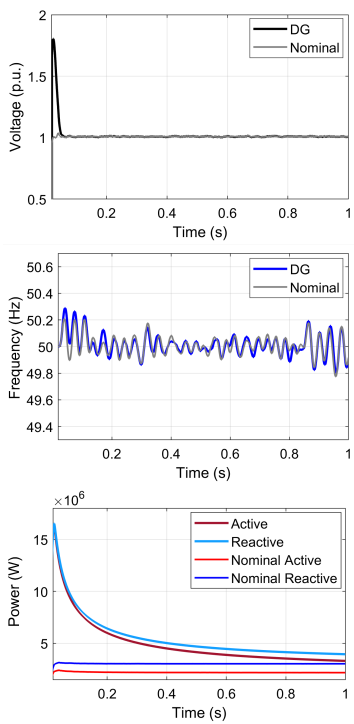

(b)
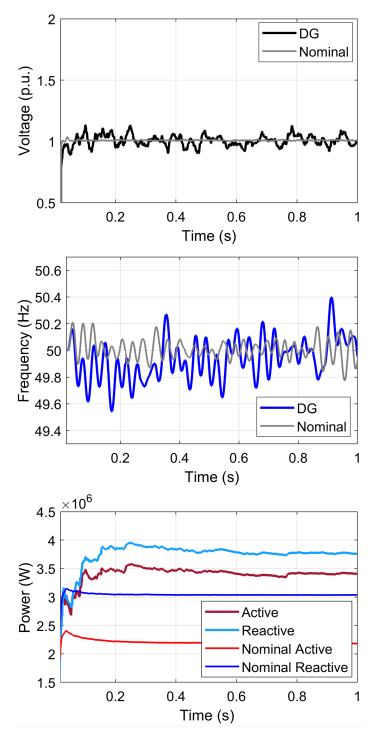

(c)
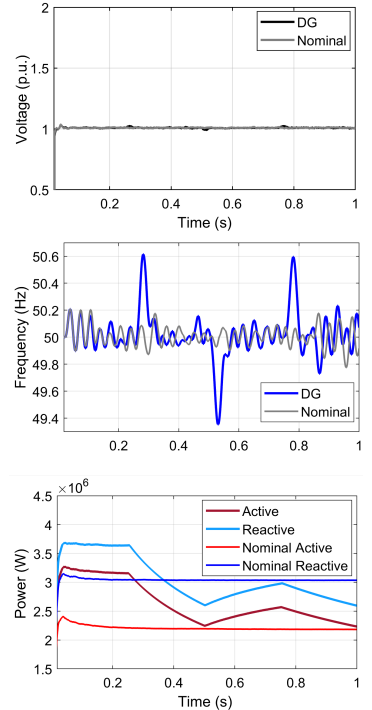

(d)

Fig. 5: Operation of inverter-based DG control under: (a) nominal conditions, (b) attack targeting the measurement vector, (c) additive attack targeting the control input, (d) load deviation attack.

this less dire scenario, every DG-related measurement is affected indicating that control attacks arise as prominent targets for adversaries aiming to disrupt power system operations.

\section{E. Attacks Targeting the Load Demand}

This attack model (Model (C) of Eq. (11)) involves a load deviation attack aiming to stealthily alter the power demand of DGs. The results in Fig. 5d validate the feasibility and importance of such attack vectors, presenting the voltage, frequency, and power deviations during the load demand attacks. In this scenario, we simulate a load deviation attack which subsequently alters the DG power demand. The load demand of a sectionalized DG system is "toggled" every $250 \mathrm{~ms}$ by $50 \%$ (nominal load is 2MVA at 0.95 power factor). Although the DG, being inverter-based and thus inertialess, immediately covers for the power demand alterations without generating significant voltage sags, the same cannot be argued for the DG frequency as seen in Fig. 5d. Such behavior could have caused equipment damage or activate load shedding protection mechanisms in order to sustain system frequency stability.

In this part, we employ the attack detection conditions of the residual-based approach to detect whether or not the system is performing nominally. Let $q=5$ (Eq. (17)), $N=1000$ (Eq. (23)), $p=5$ (Eq. (30)), and obtain the data-based realization of SKR $\mathcal{K}_{d, q}$ by Lemma 1 . If the residual signal $\mathbf{r}(k)$ differs by a margin greater than a predefined threshold $\mathbf{r}_{0}(k)=\Delta_{t h}$, the DG is considered to operate suspiciously and is isolated as a precaution. We apply Monte Carlo simulation to calculate $\Delta_{t h}$. Specifically, 5000 Monte Carlo simulations are performed under varying system perturbations, i.e., measurement vector, control input, load deviation attacks. Such random parameter and setup configurations allow to address the stochastic attack models. In Fig. 6, we demonstrate how the residuals vector of a DG, $\mathbf{r}_{\mathrm{DG}}(k)$, is affected when an additive control input

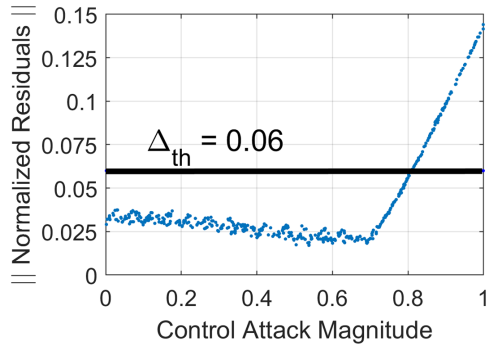

(a)

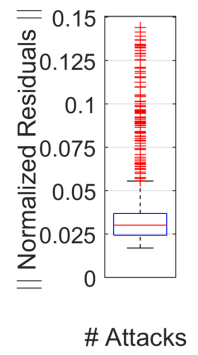

(b)
Fig. 6: Normalized residuals under additive control input attack: (a) residuals and $\Delta_{t h}$ for the islanding methodology, and (b) box plot of the residuals.

attack, as the most critical type of attack according to the previous results, is initiated. In this scenario, the DG inverter's modulation control inputs are altered. The figure presents the results of 500 simulations in which uniformly distributed control input perturbations $\mathbf{e} \in[0,1]$ are injected to the inverter controller. On the vertical axis of Fig. 6 we can observe how the residual dynamics are affected once a control input perturbation e (shown on the horizontal axis) is introduced. Based on the results depicted in Figs. 6a and 6b we select $\Delta_{t h}$ to be equal to 0.06 , which exceeds the $75^{t h}$ percentile plus the interquartile distance. This selection allows for the majority of the observations to be correctly classified, and the control attack outliers, posing the greatest danger for the inverter-based DG controller, to be effectively detected.

\section{F. Attack Detection: Residual-based Islanding}

The attack detectable conditions and calculation of the residual dynamics of the MG model $\mathbf{r}_{0}(k)=\Delta_{t h}$ can indentify a DG which is operating suspiciously and island it from the 

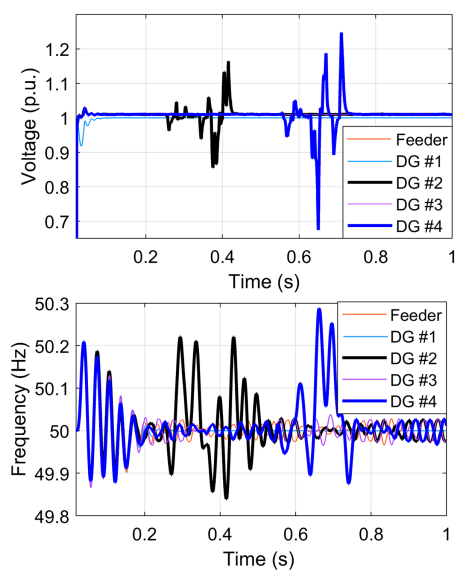

(a)
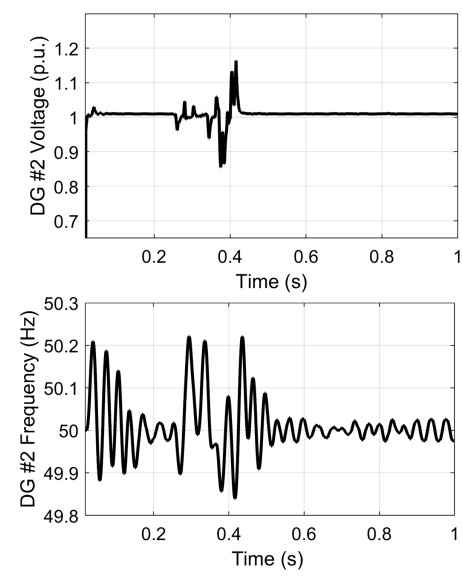

(b)
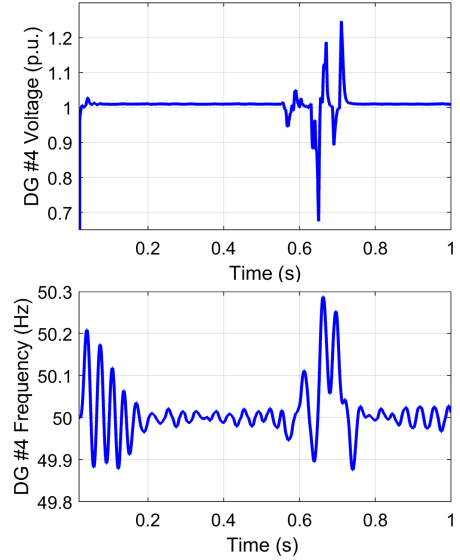

(c)

Fig. 7: Voltage and frequency measurements when DGs \#2 and \#4 are under additive control input attacks. The attack on DG \#2 is initiated at $0.25 \mathrm{~s}$ while the attack on DG \#4 starts at $0.55 \mathrm{~s}$. Voltage and frequency measurements of: (a) both DGs \#2 and \#4, (b) only DG \#2, (c) only DG \#4.

MG. In Figs. 7 and 8 , we present the results of two sequential control input attacks targeting DGs \#2 and \#4, with the voltage and frequency data being captured at buses 7 and 9 of Fig. 1 . respectively. The attack on DG \#2 is initiated at $t=0.25 \mathrm{~s}$ with a duration of $0.05 \mathrm{~s}$. Following our approach, DG \#2 is promptly disconnected from the MG due to its residual dynamics exceeding the attack-free condition $\Delta_{t h}$. A similar behavior is observed with DG \#4, in which the additive control attack occurs between $\Delta t=0.55-0.6 s$. Figs. $7 \mathrm{~b}$ and $7 \mathrm{c}$ demonstrate the inherent DG voltage and frequency before, during, and after the attack. Evidently, according to Figs. $7 \mathrm{~b}$ and $7 \mathrm{c}$, if the attack duration of each DG was prolonged, their abnormal behavior would have severely impacted the islanded MG, leading to potential instability issues which could require load shedding actions. Furthermore, in Fig. 8 we demonstrate how the reported control input attacks affect the residual signals. Their values, immediately after the attacks are initiated, at $t=0.25 \mathrm{~s}$ and $t=0.55 \mathrm{~s}$, exhibit significant deviation which triggers the islanding mechanism leading to the DG disconnection from the MG. In particular, Fig. 8a exhibits the residual behavior under an attack targeting sequentially both DG \#2 and DG \#4. Fig. 8 b shows the normalized residual deviations when DG \#2 is attacked, while Fig. 8c depicts the residual signals when DG \#4 is attacked.

\section{CONClusions And Future Work}

In this work, a novel detection strategy is proposed for islanded MG operation against disruptive cyber-physical attacks. We present three different attack models on the system measurement data, sparse additive attacks on the model inputs, and load deviation attacks on system states. We further develop the conditions to capture such attack vectors in a detection framework and simulate both the effect of the attacks as well as the detection residuals in a four inverter-based DG MG system.

Future work of this paper can be divided into three aspects. Our goal is, besides (1) the identification of tight attack detection thresholds, to (2) develop a high-level isolation logic that permits the preventive isolation of the interconnected $\mathrm{MG}$ subsystems and components with higher failure and attack probability. This segregation into sustainable minigrids will overcome the need for real-time separation decisions which could potentially cause cascading effects. In terms of the isolation logic and prevention decisions, and due to the distributed nature and the changing real-time characteristics of agent subsystems, we plan to develop severity indices to indicate: (i) whether the intentional isolation can support the network resilience or worsen it, and (ii) the formation of sustainable islanded subsystems. Such metrics will capture the system conditions during attacks as a function of impact assessment, the occurrence probability, and a subsystem reputation index. Furthermore, we plan to (3) realize the integrated design of attack models and attack-detection control to uncertain cyberphysical events which may cause parameter perturbations and changes of operating points, and extend the evaluation of the approach in real scenarios and against existing benchmarks to show the unequivocal contribution of the proposed solution.

\section{REFERENCES}

[1] A. Vasilakis et al., "The evolution of research in microgrids control," IEEE OAJPE, vol. 7, pp. 331-343, 2020.

[2] T. V. Vu et al., "Cyber-physical microgrids: Toward future resilient communities," IEEE Industrial Electronics Magazine, vol. 14, no. 3, pp. 4-17, 2020.

[3] C. Konstantinou, "Towards a secure and resilient all-renewable energy grid for smart cities," IEEE Consumer Electronics Magazine, 2021.

[4] B. Sobczak, "Experts assess damage after first cyberattack on U.S. grid," [Online]. Available: https://www.eenews.net/stories/1060281821

[5] M. R. Habibi et al., "Detection of false data injection cyber-attacks in dc microgrids based on recurrent neural networks," IEEE Journal of Emerging and Selected Topics in Power Electronics, 2020.

[6] J. James, Y. Hou, and V. O. Li, "Online false data injection attack detection with wavelet transform and deep nns," IEEE Transactions on Industrial Informatics, vol. 14, no. 7, pp. 3271-3280, 2018.

[7] B. Li et al., "Detecting false data injection attacks against power system state estimation with fast go-decomposition," IEEE Transactions on Industrial Informatics, vol. 15, no. 5, pp. 2892-2904, 2018. 


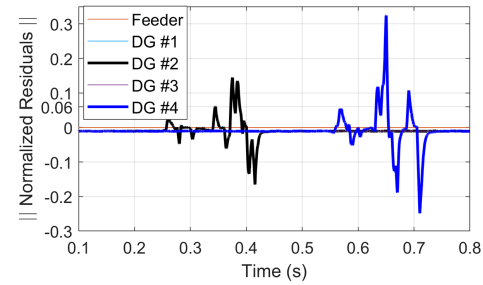

(a)

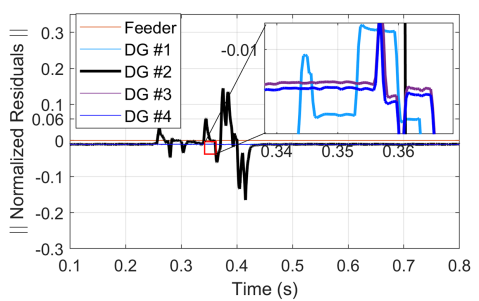

(b)

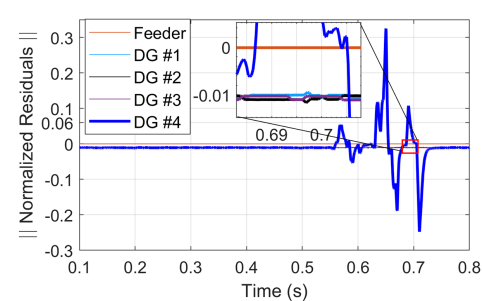

(c)

Fig. 8: Residual dynamics when DGs \#2 and \#4 are under additive control input attacks. The attack on DG \#2 starts at $0.25 \mathrm{~s}$ $\&$ the attack on DG \#4 starts at $0.55 \mathrm{~s}$. Residuals when under attack: (a) both DGs \#2 and \#4, (b) DG \#2, and (c) DG \#4.

[8] S. Ntalampiras, "Automatic identification of integrity attacks in cyberphysical systems," Expert Systems with Applications, vol. 58, pp. 164 $173,2016$.

[9] O. A. Beg, T. T. Johnson, and A. Davoudi, "Detection of false-data injection attacks in cyber-physical dc microgrids," IEEE Transactions on industrial informatics, vol. 13, no. 5, pp. 2693-2703, 2017.

[10] F. Pasqualetti, F. Dörfler, and F. Bullo, "Attack detection and identification in cyber-physical systems," IEEE transactions on automatic control, vol. 58, no. 11, pp. 2715-2729, 2013.

[11] I. Zografopoulos et al., "Cyber-physical energy systems security: Threat modeling, risk assessment, resources, metrics, and case studies," IEEE Access, vol. 9, pp. 29775-29818, 2021.

[12] Z. Huang, Y. Chen, and D. Chavarría-Miranda, "High-performance computing for real-time grid analysis and operation," in High performance computing in power and energy systems. Springer, 2013, pp. 151-188.

[13] S. Liu, X. Wang, and P. X. Liu, "Impact of communication delays on secondary frequency control in an islanded microgrid," IEEE Transactions on Industrial Electronics, vol. 62, no. 4, pp. 2021-2031, 2014.

[14] H. Zhang et al., "Distributed load sharing under false data injection attack in an inverter-based microgrid," IEEE Transactions on Industrial Electronics, vol. 66, no. 2, pp. 1543-1551, 2018.

[15] Q. Zhou et al., "A cyber-attack resilient distributed control strategy in islanded microgrids," IEEE Transactions on Smart Grid, vol. 11, no. 5, pp. 3690-3701, 2020.

[16] S. Sahoo, T. Dragičević, and F. Blaabjerg, "An event-driven resilient control strategy for dc microgrids," IEEE Transactions on Power Electronics, vol. 35, no. 12, pp. 13714-13 724, 2020.

[17] H. Fawzi, P. Tabuada, and S. Diggavi, "Secure estimation and control for cyber-physical systems under adversarial attacks," IEEE Transactions on Automatic control, vol. 59, no. 6, pp. 1454-1467, 2014.

[18] Y. Shoukry and P. Tabuada, "Event-triggered state observers for sparse sensor noise/attacks," IEEE Transactions on Automatic Control, vol. 61, no. 8, pp. 2079-2091, 2015.

[19] S. Liu et al., "Stochastic stability analysis and control of secondary frequency regulation for islanded microgrids under random denial of service attacks," IEEE Transactions on Industrial Informatics, vol. 15, no. 7, pp. 4066-4075, 2018.

[20] M. Pajic, I. Lee, and G. J. Pappas, "Attack-resilient state estimation for noisy dynamical systems," IEEE Transactions on Control of Network Systems, vol. 4, no. 1, pp. 82-92, 2017.

[21] S. X. Ding, Data-driven design of fault diagnosis and fault-tolerant control systems. Springer, 2014

[22] X. Wang et al., "Investigation of positive feedback anti-islanding control for multiple inverter-based distributed generators," IEEE Transactions on Power Systems, vol. 24, no. 2, pp. 785-795, 2008.

[23] Q. Li et al., "Networked and distributed control method with optimal power dispatch for islanded microgrids," IEEE Transactions on Industrial Electronics, vol. 64, no. 1, pp. 493-504, 2016.

[24] Z. Miao, A. Domijan, and L. Fan, "Investigation of microgrids with both inverter interfaced and direct ac-connected ders," IEEE Transactions on Power Delivery, vol. 26, no. 3, pp. 1634-1642, 2011.

[25] Y. Khayat et al., "On the secondary control architectures of ac microgrids: An overview," IEEE Transactions on Power Electronics, vol. 35, no. 6, pp. 6482-6500, 2020.

[26] X. Wang, Investigation of positive feedback anti-islanding scheme for inverter-based distributed generation. ProQuest, 2009.

[27] J. Valappil and C. Georgakis, "Systematic estimation of state noise statistics for extended kalman filters," AIChE Journal, vol. 46, no. 2 pp. 292-308, 2000 .
[28] S. Sahoo et al., "A stealth cyber attack detection strategy for dc microgrids," IEEE Transactions on Power Electronics, 2018.

[29] A. Sargolzaei et al., "Detection and mitigation of false data injection attacks in networked control systems," IEEE Transactions on Industrial Informatics, vol. 16, no. 6, pp. 4281-4292, 2020.

[30] G. Liang et al., "A review of false data injection attacks against modern power systems," IEEE Transactions on Smart Grid, vol. 8, no. 4, pp. $1630-1638,2016$

[31] S. Liu, P. X. Liu, and X. Wang, "Effects of cyber attacks on islanded microgrid frequency control," in IEEE 20th Int'l Conf. on Computer Supported Cooperative Work in Design. IEEE, 2016, pp. 461-464.

[32] J. Kim et al., "Detection of sensor attack and resilient state estimation for uniformly observable nonlinear systems," in 2016 IEEE 55th Conference on Decision and Control (CDC). IEEE, 2016, pp. 1297-1302.

[33] S. Z. Yong, M. Zhu, and E. Frazzoli, "Switching and data injection attacks on stochastic cyber-physical systems: Modeling, resilient estimation, and attack mitigation," ACM Transactions on Cyber-Physical Systems, vol. 2, no. 2, pp. 1-2, 2018.

[34] M. Esmalifalak et al., "Stealth false data injection using independent component analysis in smart grid," in IEEE Int'l Conf. on Smart Grid Communications (SmartGridComm). IEEE, 2011, pp. 244-248.

[35] Z.-H. Yu and W.-L. Chin, "Blind false data injection attack using pca approximation method in smart grid," IEEE Transactions on Smart Grid, vol. 6, no. 3, pp. 1219-1226, 2015.

[36] A. Abbaspour et al., "Resilient control design for load frequency control system under false data injection attacks," IEEE Transactions on Industrial Electronics, 2019.

[37] Z. Zhang, Y. Wang, and L. Xie, "A novel data integrity attack detection algorithm based on improved grey relational analysis," IEEE Access, vol. 6, pp. 73423-73433, 2018.

[38] S. Lakshminarayana, S. Adhikari, and C. Maple, "Analysis of iot-based load altering attacks against power grids using the theory of secondorder dynamical systems," IEEE Transactions on Smart Grid, 2021.

[39] J. Ospina et al., "On the feasibility of load-changing attacks in power systems during the covid-19 pandemic," IEEE Access, vol. 9, pp. 2545 2563, 2021.

[40] S. Sahoo, T. Dragičević, and F. Blaabjerg, "Cyber security in control of grid-tied power electronic converters-challenges and vulnerabilities," IEEE Journal of Emerging\&Selected Topics in Power Electronics, 2019.

[41] Q. Shafiee, J. M. Guerrero, and J. C. Vasquez, "Distributed secondary control for islanded microgrids-a novel approach," IEEE Transactions on Power Electronics, vol. 29, no. 2, pp. 1018-1031, 2014.

[42] M. G. Taul et al., "Systematic approach for transient stability evaluation of grid-tied converters during power system faults," in 2019 IEEE Energy Conversion Congress and Exposition (ECCE), 2019, pp. 5191-5198.

[43] M. Eskandari and A. V. Savkin, "On the impact of fault ride-through on transient stability of autonomous microgrids: Nonlinear analysis and solution," IEEE Transactions on Smart Grid, vol. 12, no. 2, pp. 9991010, 2021.

[44] X. He and H. Geng, "Transient stability of power systems integrated with inverter-based generation," IEEE Transactions on Power Systems, vol. 36, no. 1, pp. 553-556, 2021.

[45] M. H. Ghaderi, N. Rashidirad, and M. Hamzeh, "Analyzing delaypower relationship of the grid-connected inverters in system small signal stability," in 2019 10th International Power Electronics, Drive Systems and Technologies Conference (PEDSTC), 2019, pp. 445-449.

[46] S. Hadavi et al., "Small signal stability assessment of islanded vsi-based microgrids considering load variations," in 2014 IEEE Conference on Energy Conversion (CENCON), 2014, pp. 440-444. 